\title{
CCL-34, a synthetic toll-like receptor 4 activator, modulates differentiation and maturation of myeloid dendritic cells
}

\author{
Shu-Ling Fu ${ }^{1}$, Chun-Cheng Lin ${ }^{2}$, Ming-Ling Hsu ${ }^{3}$, Sheng-Hung Liu ${ }^{1}$, Yu-Chuen Huang ${ }^{4}$, \\ Yu-Jen Chen ${ }^{1,3,5}$ \\ ${ }^{1}$ Institute of Traditional Medicine, National Yang-Ming University, Taipei, Taiwan \\ ${ }^{2}$ Department of Chemistry, National Tsing Hua University, Hsinchu, Taiwan \\ ${ }^{3}$ Department of Medical Research, MacKay Memorial Hospital, Taipei, Taiwan \\ ${ }^{4}$ School of Chinese Medicine, College of Chinese Medicine, China Medical University, Taichung, Taiwan \\ ${ }^{5}$ Department of Radiation Oncology, MacKay Memorial Hospital, Taipei, Taiwan \\ Correspondence to: Yu-Jen Chen, e-mail: chenmdphd@gmail.com
}

Keywords: CCL-34, TLR4, dendritic cell, differentiation, maturation

Received: June 21, 2015

Accepted: January 26, 2016

Published: February 11, 2016

\section{ABSTRACT}

CCL-34, a synthetic a-galactosylceramide analog, has been reported as an activator of toll-like receptor 4 (TLR4) in macrophages. TLR4 is highly expressed in dendritic cell (DC) and several TLR4 agonists are known to trigger DC maturation. We herein evaluated the effect of CCL-34 on DC maturation. Human CD14 ${ }^{+}$monocyte-derived immature DC were treated with CCL-34, its inactive structural analog CCL-44, or LPS to assess the DC maturation. CCL-34 induced DC maturation according to their characteristically dendrite-forming morphology, CD83 expression and IL-12p70 production. The allostimulatory activity of $D C$ on proliferation of naive $C D 4{ }^{+} C D 45^{+} R^{+}$ $T$ cells and their secretion of interferon- $\gamma$ was increased by CCL-34. Phagocytosis, an important function of immature DC, was reduced after CCL-34 treatment. All these effects related to DC maturation were evidently induced by positive control LPS but not by CCL-44 treatment. TLR4 neutralization impaired human DC maturation triggered by CCL-34. The induction of IL-12, a hallmark of DC maturation, by CCL-34 and LPS was only evident in TLR4-competent $\mathrm{C} 3 \mathrm{H} / \mathrm{HeN}$, but not in TLR4-defective $\mathrm{C} 3 \mathrm{H} / \mathrm{HeJ}$ mice. CCL-34 could further elicit the antigen presentation capability in mice inoculated with doxorubicin-treated colorectal cancer cells. In summary, CCL-34 triggers DC maturation via a TLR4-dependent manner, which supports its potential application as an immunostimulator.

\section{INTRODUCTION}

DC are specialized leukocytes that present antigens to naive $\mathrm{T}$ cells, thus playing a pivotal role in bridging cell-mediated and humoral immune responses in vivo [1]. The ability of DC to stimulate T cells is mainly attributed to their ability to capture antigens, migrate into lymphoid organs, and express high levels of immunostimulatory molecules, such as major histocompatibility complex (MHC) class II, B7.1 (CD80), B7.2 (CD86), and IL-12 [1]. Upon exposure to various microbial and inflammatory products (e.g., lipopolysaccharide [LPS], interleukin-1 [IL-1], tumor necrosis factor- $\alpha[$ TNF- $\alpha]$ ), DC matures and migrates into lymphoid tissues to interact with $\mathrm{T}$ and $\mathrm{B}$ cells [2-5].
Toll-like receptor 4 (TLR4) activation on antigen presenting cells (APCs) can enhance immune responses to antigens and augment the effectiveness of vaccines [6-8]. LPS, the natural TLR4 agonist, causes marked inflammatory responses with major safety consideration for clinical use. Thus, development of novel TLR4 activators to potentiate adaptive immune responses without causing strong inflammation remains an important task [9]. Glycolipid-based TLR4 agonists, such as monophosphoryl lipid A (MPL) and RC-529, have been successfully developed as adjuvant for vaccination. These agents are potent TLR4 activators with better toxicity profile compared with LPS [10].

CCL-34, a synthetic bioactive glycolipid developed previously from our research team, has been reported as 
a TLR4 activator, promoting macrophage activation and macrophage-mediated cytotoxicity of cancer cells $[11,12]$. In a syngeneic bladder cancer cell model, CCL-34 was demonstrated to delay tumor growth via TLR4-dependent activation of immune cells [12]. The tumor sizes in TLR4-defective mice after CCL-34 treatment are close to those in vehicle-treated group, indicating that TLR4 is the main molecular target of CCL-34. Since several TLR4 agonists, such as LPS, are known to be capable of trigger DC maturation, we aimed to evaluate whether CCL34 can promote DC maturation. We used human monocyte-derived immature DC to examine the effect of CCL-34 on their maturation by assessing the morphology, phenotype, cytokine production, stimulation of allogeneic naive T cells. The dependence of CCL-34induced DC maturation on TLR4 was also demonstrated. Furthermore, the effect of CCL-34 on antigen presentation in vivo was analyzed.

\section{RESULTS}

\section{Morphological changes}

Immature DC collected on day 7 before maturation showed round contours without evident dendrites (Figure 1A). The cytokine- and LPS-triggered DC on day 7 had morphological characteristics typical of mature
DC, including being non-adherent and having multiple cytoplasmic projections and abundant cytoplasm (Figure 1B-1C). The majority of CCL-34-treated DC manifested similar characteristic features of mature DC, indicating an activity resembling known DC maturation inducers (Figure 1D). By contrast, treatment with CCL-44, an inactive analog of CCL-34, resulted in DC showing less maturation morphology (Figure 1E).

\section{Effect of CCL-34 on recovery rate of DC}

When CCL-34 was added into culture of immature DC to trigger maturation, there was no significant effect on recovery rate of $\mathrm{DC}$, as measured by trypan blue exclusion test (Figure 2A). Evaluation of cytotoxicity using 7-AAD staining showed that CCL-44, but not CCL-34, induced cell death in an extent greater than LPS (Figure 2B).

\section{The effect of CCL-34 on DC surface marker expression}

CCL-34, but not CCL-44, induced markedly expression of CD83 on DC in a dose-dependent manner, indicating a maturation state similar to that triggered by LPS and cytokine cocktail with a lower extent (Figure 3). Since the starting cells were immature DC derived from monocytes, the expression of surface markers related to DC differentiation including CD14, CD1a, co-stimulatory
A Untreated immature DC

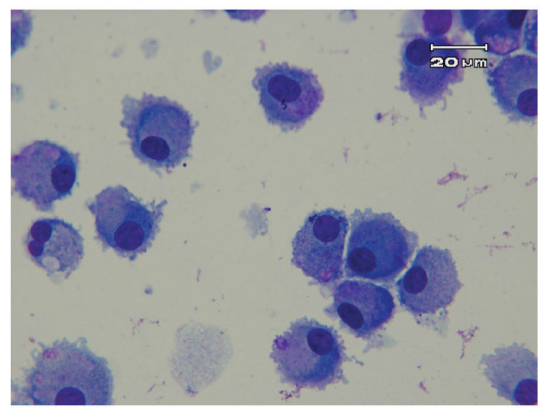

D CCL34- triggered mature DC

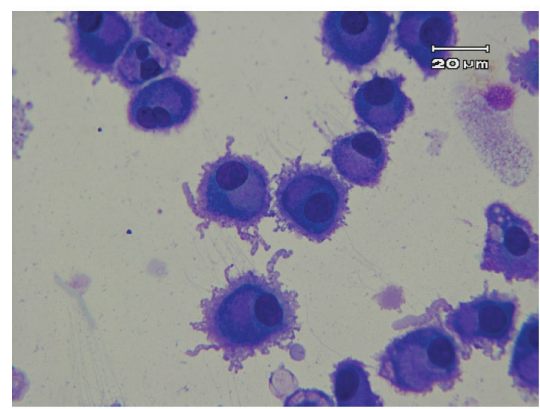

B Cytokine-triggered mature DC

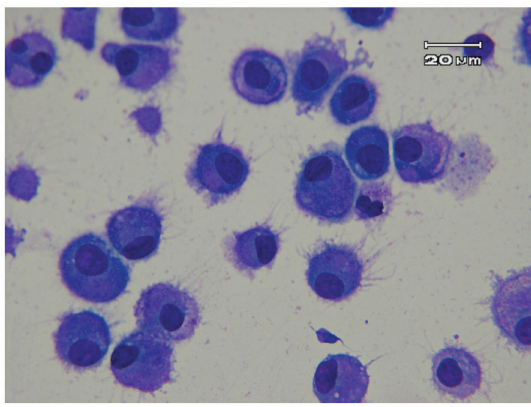

E CCL44-triggered mature DC

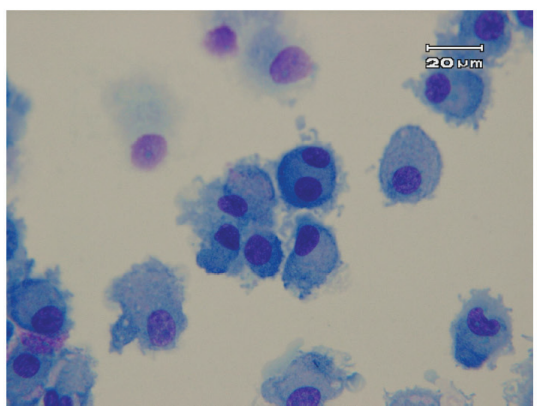

C LPS-triggered mature DC

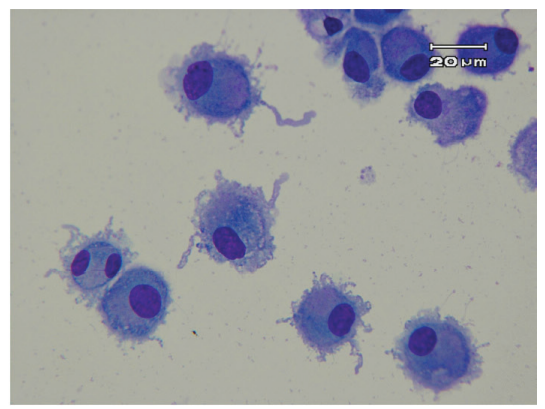

Figure 1: Morphology of monocyte-derived DC. (A) control immature DC; (B) cytokine-triggered mature DC; (C) LPS-triggered mature DC; (D) CCL-34-triggered mature DC; (E) CCL-44-treated DC. Cells were centrifuged onto microscope slides and stained with Liu's solution. Magnification for photograph is $1000 \times$. 
molecules CD80 and CD86, MHC class II molecule HLA-DR, and DC-SIGN were not significantly altered by either CCL-34 or CCL-44 (Figure 3).

\section{Secretion of IL-12p70 from DC developed in the presence of CCL-34}

As demonstrated in Figure 4, IL-12p70 levels produced by DC in the presence of CCL-34 were dosedependently increased.

\section{The effect of CCL-34 on the capacity of DC to stimulate allogeneic naive $T$ cells}

CCL-34, but not CCL-44, possessed a trend to augment the allostimulatory activity of DC on stimulating proliferation of naive $\mathrm{CD} 4^{+} \mathrm{CD} 45^{+} \mathrm{RA}^{+} \mathrm{T}$ cells (Figure 5A). IFN- $\gamma$ production by these allogeneic naive $\mathrm{T}$ cells also had a trend to be enhanced by DC differentiated in the presence of CCL-34, but not CCL-44 (Figure 5B).

\section{The effect of CCL-34 on phagocytosis of DC}

Upon stimulation of immature DC toward maturation, the phagocytic capacity is reduced. Our results demonstrated that CCL-34, but not CCL-44, reduced the phagocytosis capacity of DC, suggesting a process toward maturation (Figure 6).

\section{The effect of CCL-34 on DC is TLR4-dependent}

Because CCL-34 has been reported as a TLR4 activator in our previous work, we next evaluated whether the effect of CCL-34 on DC maturation is dependent on TLR4. For human myeloid DC, TLR4 neutralizing antibody abolished the maturation effect of CCL-34 and LPS in terms of CD83 expression (Figure 7A). To further elucidate the role of TLR4 on DC maturation triggered by CCL-34, we isolated DC precursors from TLR4competent $\mathrm{C} 3 \mathrm{H} / \mathrm{HeN}$ or TLR4-defective $\mathrm{C} 3 \mathrm{H} / \mathrm{HeJ}$ mice and subjected to differentiation induction toward DC. By measurement of the IL-12 production, it was found

A

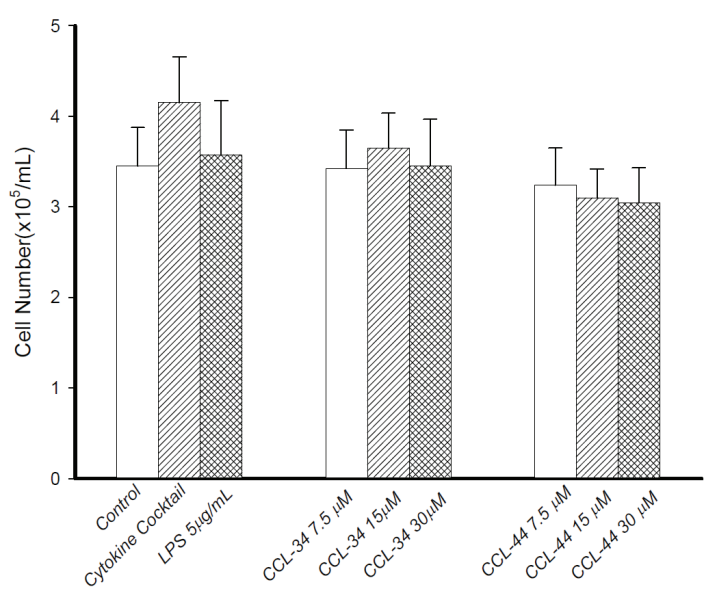

B

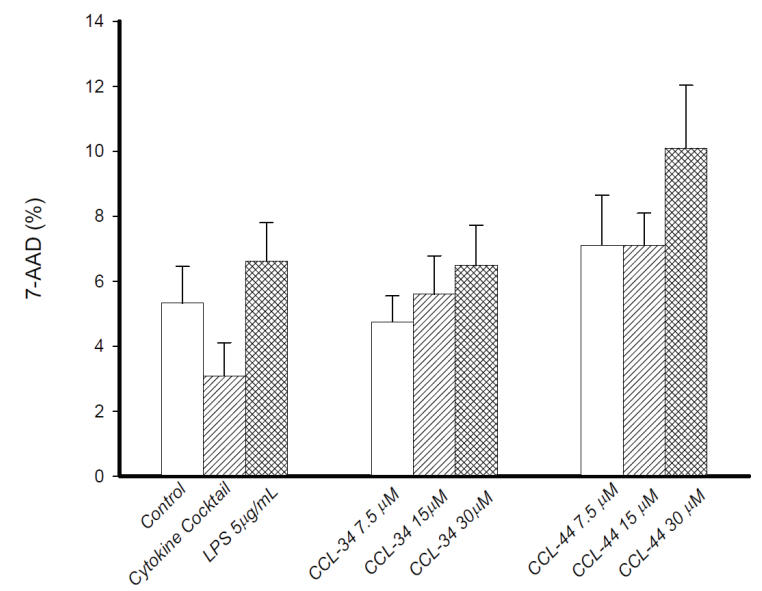

Figure 2: Effect of CCL-34 and CCL-44 on vaibility and cytotoxicity of DC. (A) Number of viable DC recovered from starting CD14 ${ }^{+}$monocytes was estimated by trypan blue exclusion test. (B) Cytotoxicity of CCL-34 and CCL-44 on DC was assessed by 7-AAD staining and flow cytometry. Data from 5 separate experiments by using monocytes from 5 donors are expressed as mean \pm SEM. 


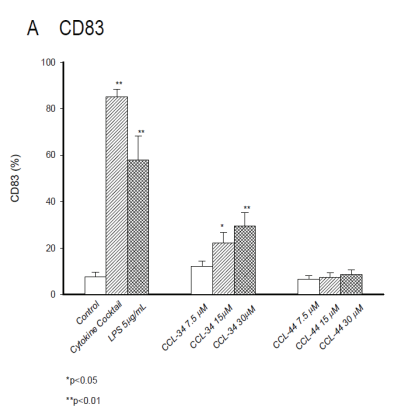

E CD86

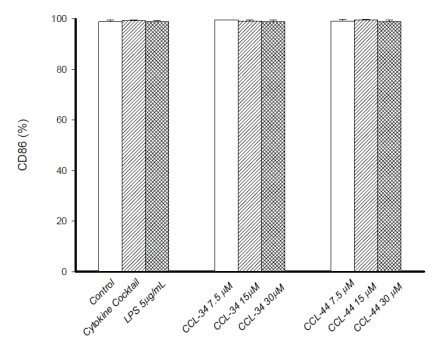

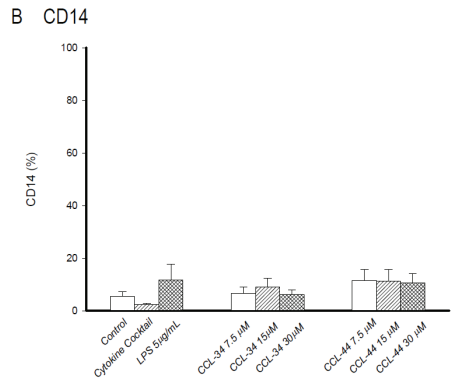

F DC-SIGN

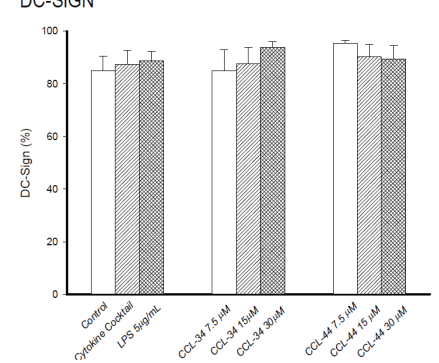

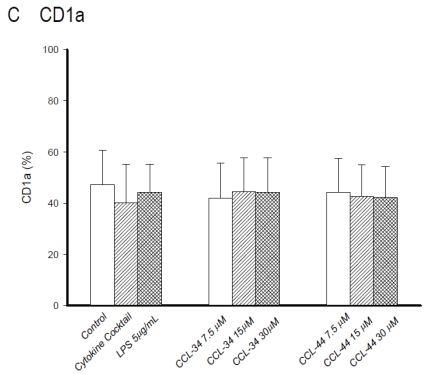

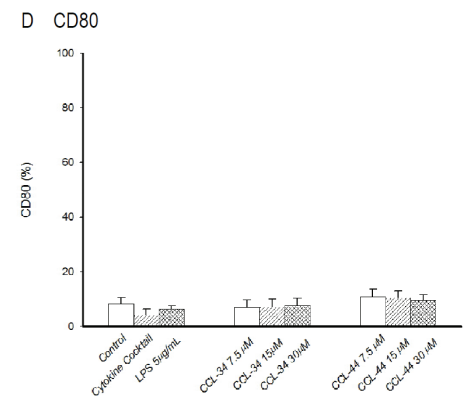

G HLA-DR

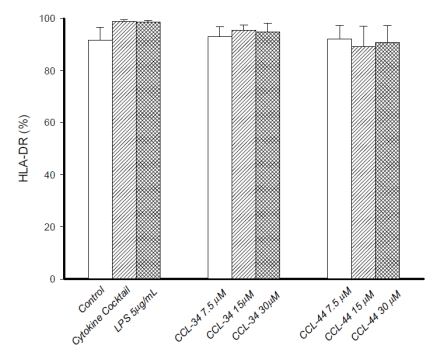

Figure 3: Expression of surface molecules on monocyte-derived DC. (A) CD83. (B) CD14. (C) CD1a. (D) CD80. (E) CD86. (F) DC-SIGN. (G) HLA-DR. Data from 5 separate experiments by using monocytes from 5 donors are expressed as mean \pm SEM.

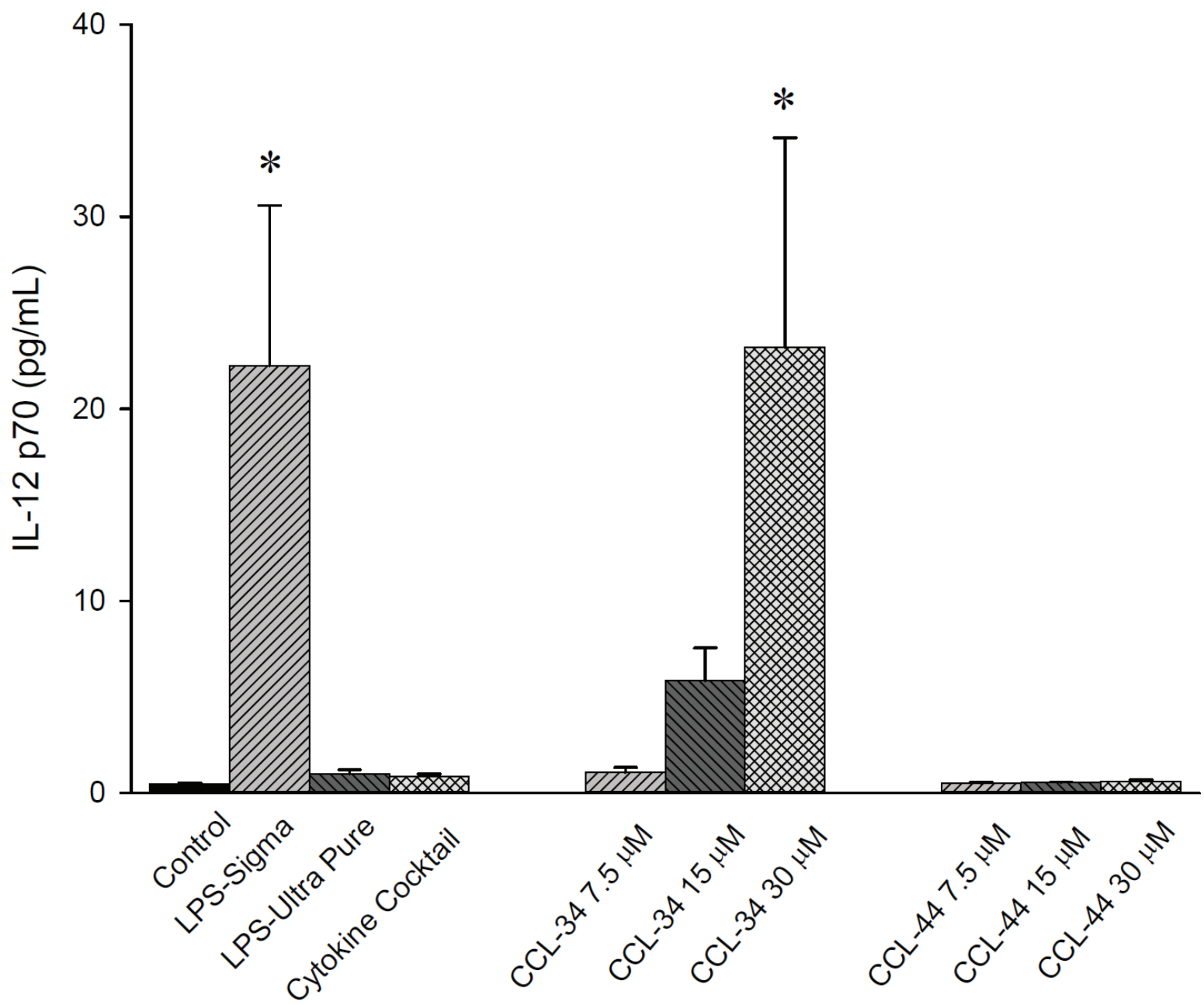

Figure 4: IL-12 production by human myeloid DC. Data from 5 to 7 separate experiments are expressed as mean \pm SEM. * $p$-value $<0.05$ compared with control group. 
that mice DC maturation triggered by CCL-34 and LPS was only evident in TLR4-competent $\mathrm{C} 3 \mathrm{H} / \mathrm{HeN}$, but not in TLR4-defective $\mathrm{C} 3 \mathrm{H} / \mathrm{HeJ}$ mice (Figure 7B). Taken together, the activity of CCL-34 on triggering DC maturation is TLR4-dependent.

\section{The effect of CCL-34 on DC antigen presentation in vivo}

As demonstrated in Figure 8, the amount of antigenspecific CTL in tumor draining lymph nodes of CCL-34treated mice was greater than those of vehicle and CCL-44, indicating a structure-specific DC immunity in vivo.

\section{DISCUSSION}

We here demonstrated that CCL-34, a bioactive synthetic glycolipid developed previously by our team, could trigger DC maturation via a TLR4-dependent manner. The TLR4 activators reported to elicit immunity of DC include glucopyranosyl lipid A [13], C. nebularis lectin [14], OM-174 [15], OK-432 [16] and poly-gamma-glutamate [17]. Among currently identified TLR4 activators, CCL-34 is advantageous due to its defined structure and well-established synthesis procedure [18]. Given that CCL34 could activate TLR4 to promote macrophage function [11] and DC maturation, CCL34 may serve as a potential immune modulator for immunotherapy, such as adjuvant for vaccines.

Our previous data demonstrate that CCL-34-activated macrophages are M1-polarized based on their gene expression profiles [12]. The antitumor immunity induced by CCL-34 in vivo, including the expression of IFN- $\gamma$ and IL-12 as well as the leukocyte markers (CD11b, CD11c, CD4 and CD8), further indicated that

\section{A Allogeneic naïve T cell proliferation}
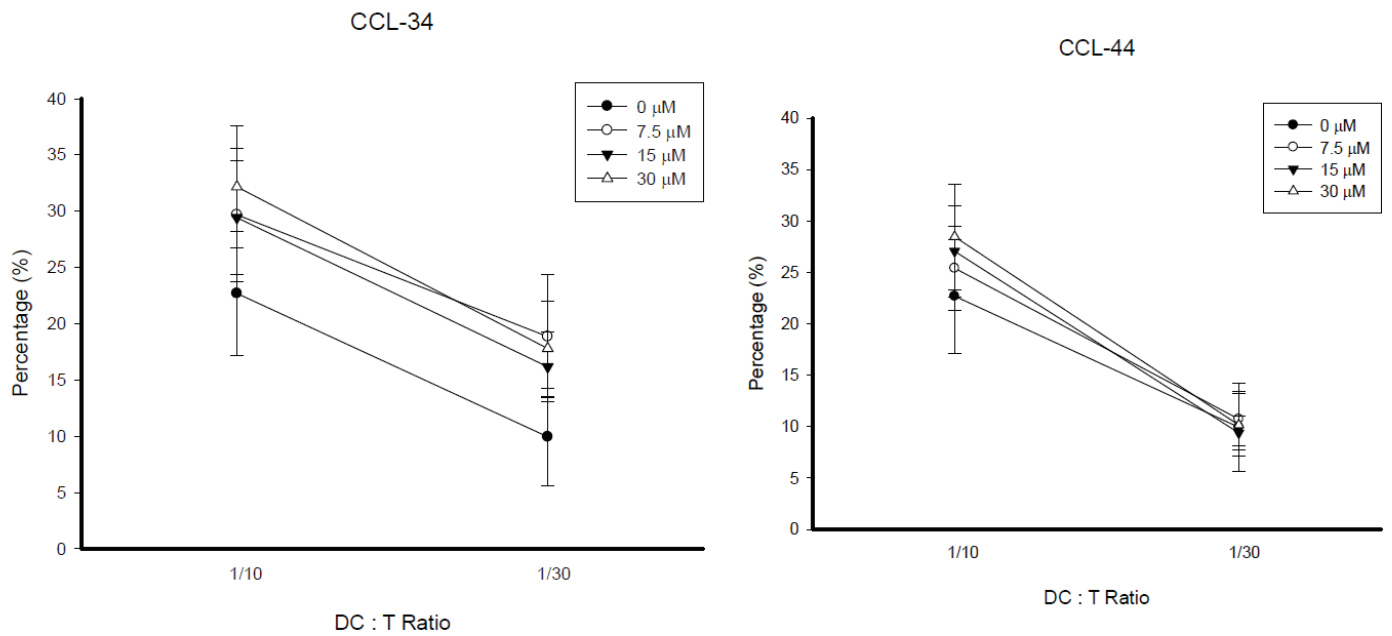

B IFN- $\gamma$ secretion by allostimulation
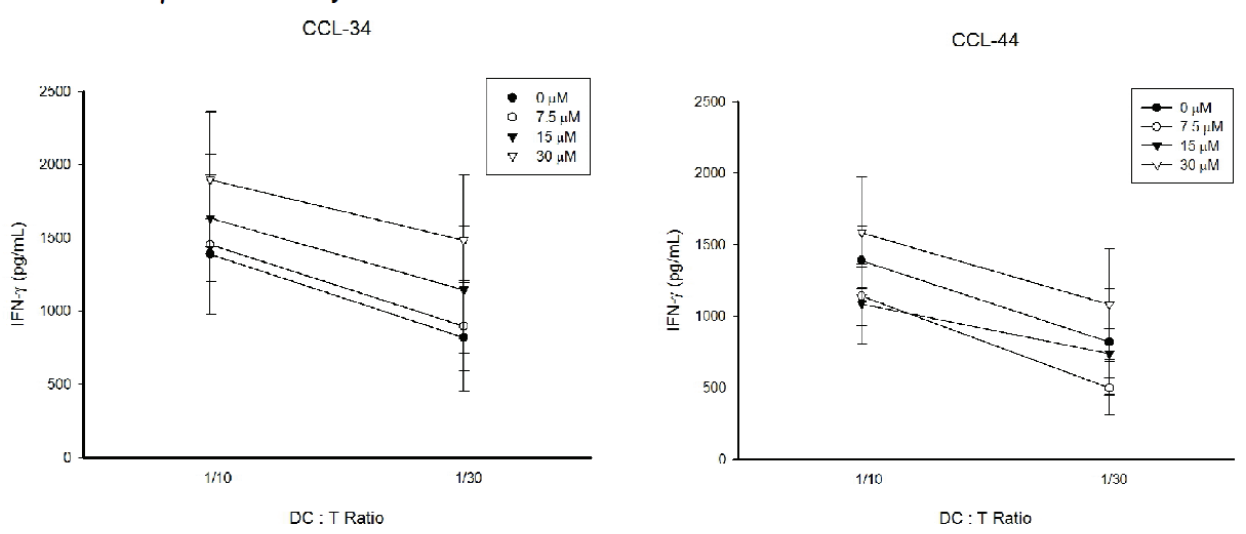

Figure 5: Proliferation and IFN- $\gamma$ secretion of allogeneic $\mathrm{CD4}^{+} \mathrm{CD} 45 \mathrm{RA}^{+}$naive $\mathrm{T}$ cell stimulated by mature $\mathrm{DC}$. The $\mathrm{CD}^{+} \mathrm{CD} 45 \mathrm{RA}^{+}$naïve T cells were isolated by using a MiniMACS system with magnetic Abs by a negative selection technique. Monocytederived DC were harvested and irradiated (3,000 cGy) with $6 \mathrm{MeV}$ x-ray. Irradiated DC were incubated with $1 \times 10^{6}$ allogeneic naive T cells at ratios of $1: 10$ or 1:30 for 5 days, after which $10 \mu \mathrm{M}$ BrdU was added to T cell cultures for $18 \mathrm{~h}$. (A) Proliferation. The cells were then collected and the incorporated BrdU was detected using flow cytometry. (B) IFN- $\gamma$ secretion. Data from 5 separate experiments are expressed as mean $\pm \mathrm{SEM}$. 


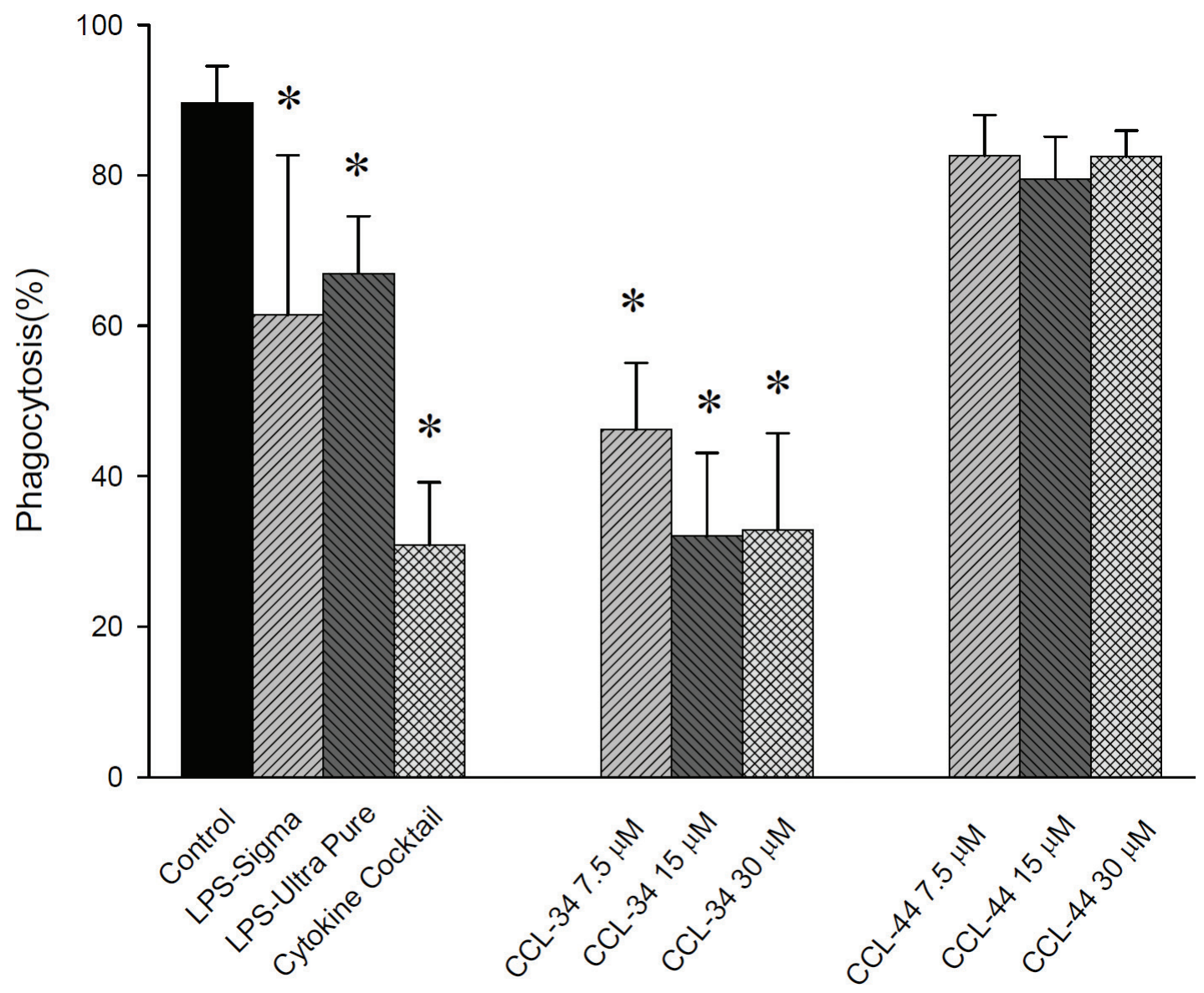

Figure 6: The effect of CCL-34 and CCL-44 on phagocytosis capacity of DC. Data from 5 separate experiments by using monocytes from 5 donors are expressed as mean \pm SEM. * $p$-value $<0.05$ compared with control group.

A

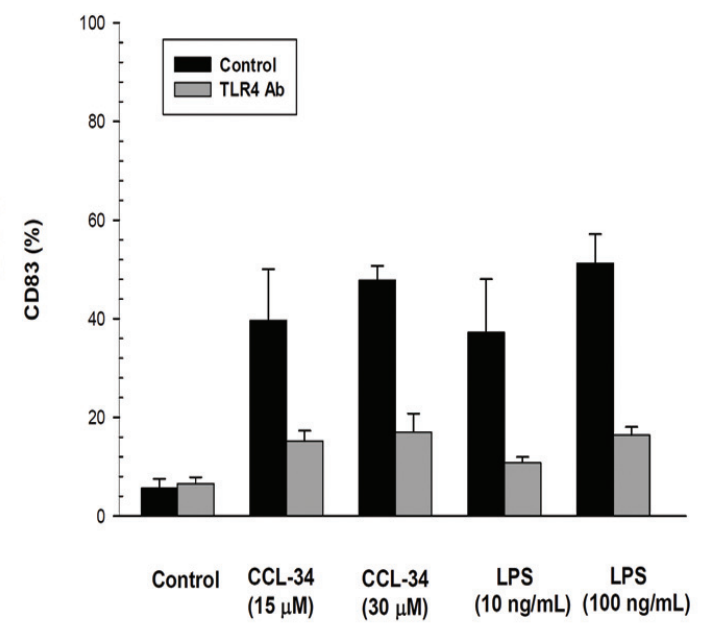

B

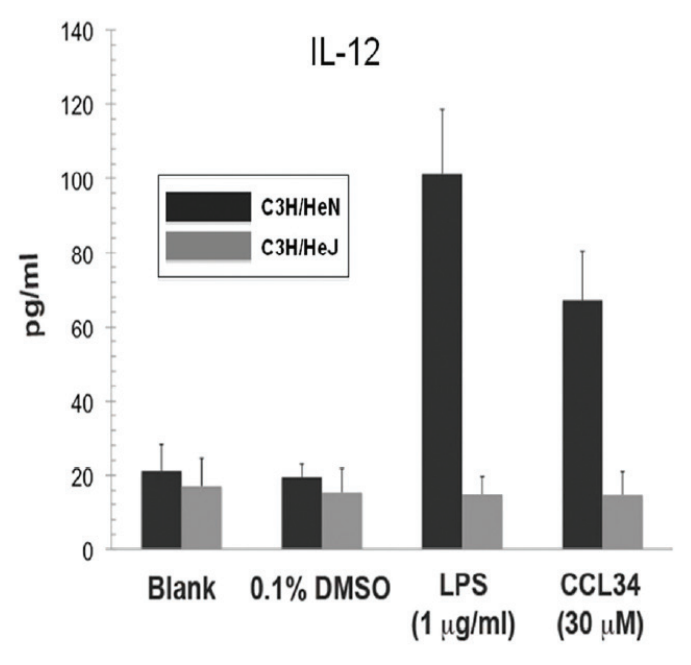

Figure 7: The effect of CCL-34 on DC maturation is TLR4-dependent. (A) CD83 expression of human DC derived from monocytes of donors with or without TLR-4 antibody blocking. (B) IL-12 production by bone marrow-derived immature DC prepared from the TLR4-defective $\mathrm{C} 3 \mathrm{H} / \mathrm{HeJ}$ and wild-type $\mathrm{C} 3 \mathrm{H} / \mathrm{HeN}$ mice (5 mice in each group). Data are expressed as mean $\pm \mathrm{SEM}$. 
Th1 adaptive immune responses are activated by CCL34 [12]. Although the CCL34-induced signaling pathway downstream TLR4 has been elucidated in macrophages $[11,12,19]$, it remains not clarified in immature DC. This issue could be addressed further in the disease model using both in vitro and in vivo experiments.

The role of TLR4 signaling in cancer has been considered a double-edged sword. Activation of TLR4 on immune cells can enhance anti-tumor immunity. However, chronic inflammation related to TLR4 activation remains as a major risk factor in cancer development [20]. Expression of TLR4 and its signaling of tumor cells have been demonstrated to induce the synthesis of soluble immune mediators that could help the tumor to invade the immune attack [21]. In our previous investigation, CCL-34 treatment suppressed tumor growth and benefited the survival in a TLR4-dependent manner [12]. It suggests that CCL-34 may have a potential as an immunotherapeutic agent against cancer in vivo without major concern for impairment of immune evasion. Furthermore, CCL-34treated mice showed induction of IL-12 and elevation of CD11c marker in tumor areas, implying the involvement of dendritic cells in the anticancer activity of CCL-34 in vivo [12]. In this study, we provide direct evidence

\section{A}
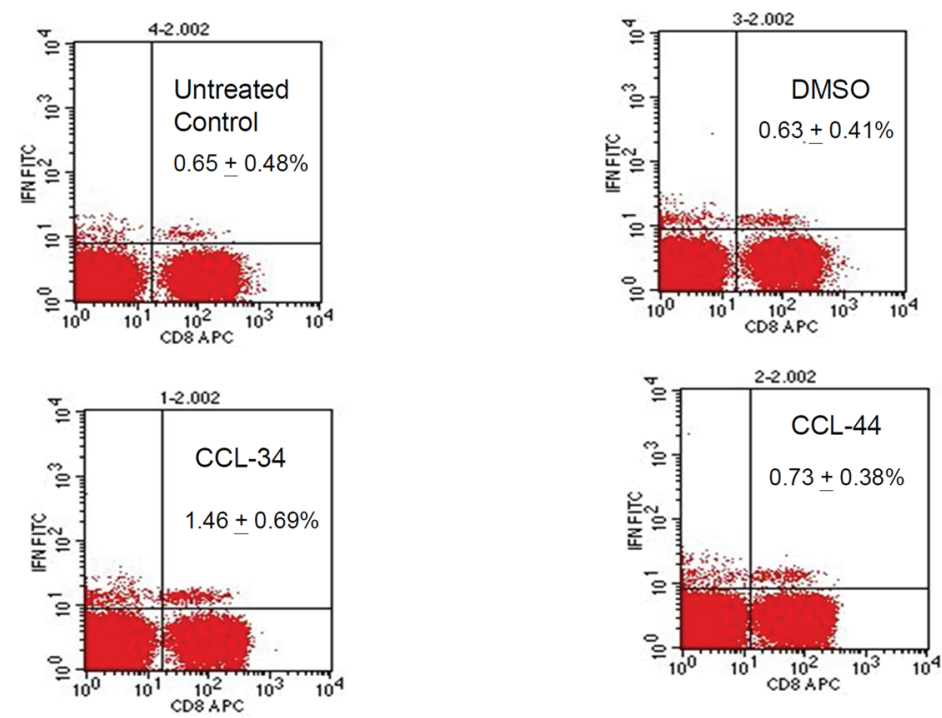

B

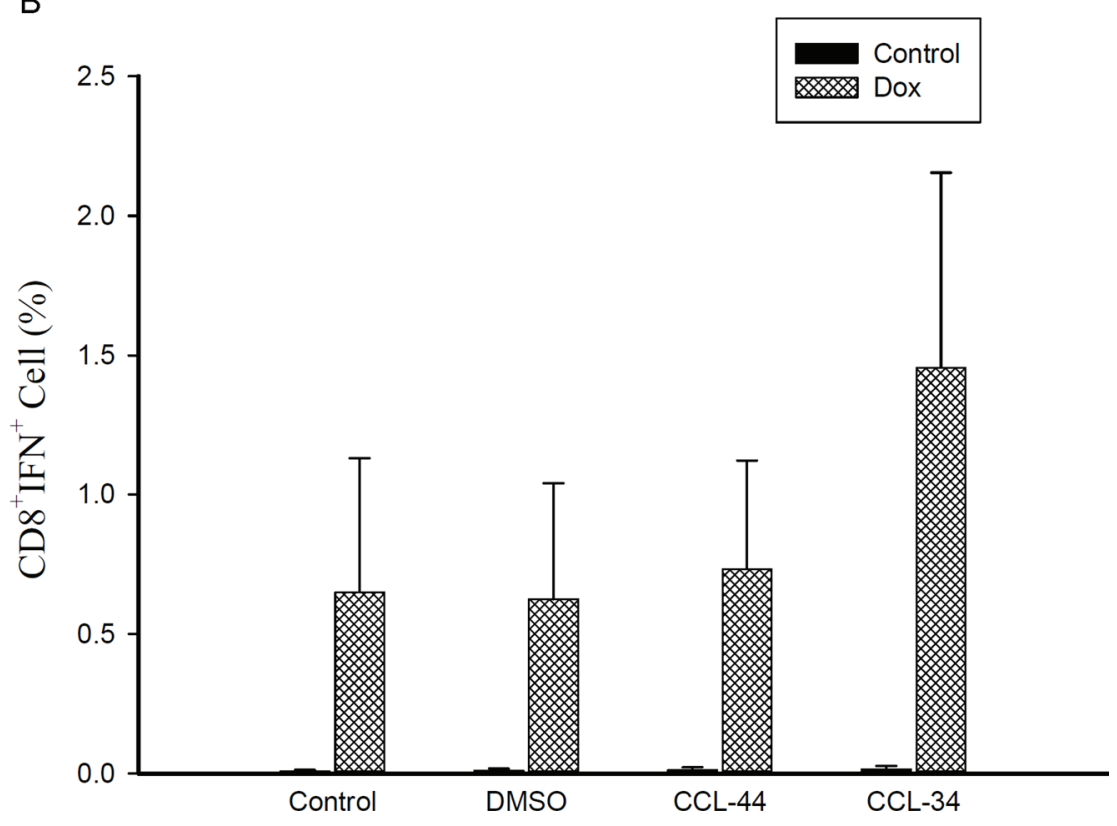

Figure 8: Flow cytometry analysis of IFN- $\gamma$-secreting antigen-specific $\mathrm{CD8}^{+}$cell in mice challenged by $\mathrm{CT26}$ cell-associated antigen. (A) representative flow cytometry data showing the percentage of Ag-specific IFN- $\gamma^{+} \mathrm{CD} 8^{+} \mathrm{T}$ cells in the tumor draining lymph nodes. (B) percentage of IFN $-\gamma^{+} \mathrm{CD} 8^{+} \mathrm{T}$ cells from each group without (black columns) or with (shaded columns) stimulation by the doxorubicin-treated tumor lysates. Data from 3 separate experiments by using monocytes from 3 donors are expressed as mean \pm SEM. 
demonstrating that CCL-34 can promote DC maturation, further supporting the idea that activation of dendritic cells by CCL-34 is crucial for its anticancer activity in vivo.

Our previous structure-activity relationship analysis of CCL-34 and its analogs revealed that both the sugar moiety and the lengths of the lipid chains are essential for its TLR4-activation activity [11, 18]. In addition, CCL-34 is an optimal TLR4-activating compound among tested structures $[12,18]$. It was previously shown that CCL-34 has low toxicity to primary bone marrow cells and its safety has been examined in tumor-bearing mice without major organ toxicity: CCL-34 treatment (i.p. $100 \mu \mathrm{g} /$ mice, every 3 4 day in a 21-day treatment) did not significantly decrease body weight, nor affect liver and kidney functions based on serum biochemistry data ([12] and Supplementary data). Based on the safety and the multiple bioactivities we demonstrated in this and previous studies, we think that CCL-34 is a TLR-4 activating immune stimulator meriting further exploration of its potential clinical application.

\section{MATERIALS AND METHODS}

\section{Generation of human dendritic cells}

Human peripheral blood mononuclear cells were obtained from healthy donors using Histopaque (Amersham Pharmacia Biotech, Piscataway, NJ, USA) density gradient centrifugation. Erythrocytes were lysed by treatment with $0.9 \%$ ammonium chloride for $3 \mathrm{~min}$ at $37^{\circ} \mathrm{C}$. Subsequently, CD $14^{+}$cells were purified by high-gradient magnetic sorting using the miniMACS system with anti-CD14 microbeads (Miltenyi Biotec, Bergisch Bladbach, Germany). After $2 \mathrm{~h}$ incubation at $37^{\circ} \mathrm{C}$, nonadherent cells were removed and adherent cells were collected. The purity of isolated $\mathrm{CD} 14^{+}$ monocytes was over $90 \%$ on flow cytometric analysis. Immature $\mathrm{DC}$ were generated from $\mathrm{CD} 14^{+}$monocytes by culture in RPMI 1640 medium supplemented with $10 \%$ fetal calf serum, $100 \mathrm{ng} / \mathrm{mL}$ granulocyte-macrophage colony-stimulating factor (GM-CSF) (Schering-Plough, Munich, Germany) and $50 \mathrm{ng} / \mathrm{mL}$ IL-4 (R \& D Systems, Minneapolis, MN, USA) every 3 days for 6 days in a humidified $5 \% \mathrm{CO}_{2}$ incubator. To trigger maturation of DC, immature DC were incubated with LPS, CCL-34 $(0,7.5,15,30 \mathrm{mM})$, and combination of pro-inflammatory cytokines including $5 \mathrm{ng} / \mathrm{mL}$ TNF- $\alpha, 5 \mathrm{ng} / \mathrm{mL}$ IL- $1 \beta$, $15 \mathrm{ng} / \mathrm{mL}$ IL-6 (R \& D Systems, Minneapolis, MN) and $1 \mu \mathrm{g} / \mathrm{mL}$ prostaglandin E2 (PGE2) (Sigma-Aldrich, St. Louis, MO, USA) for a further $24 \mathrm{~h}$. To examine the effect of LPS as a positive control, we tested two kinds of LPS to select an appropriate stimulator for DC maturation. As shown in Figure 4, LPS, but not LPS-Ultra pure, could stimulate IL-12p70 production from DC. Although not statistically significant, the mean phagocytosis percentage of DC in LPS group was smaller than in LPS-Ultra pure group, implicating a more potent maturation induction by LPS. According to these data, we selected LPS as positive control thereafter. It has been shown that monocyteconditioned medium containing a combination of pro-inflammatory cytokines (i.e. TNF- $\alpha$, IL-1, IL-6 and PGE2) triggers efficient DC maturation. Thus, we used a cocktail of these cytokines with defined concentrations that had also been applied by other researchers for this purpose. CCL-34 and CCL-44 were synthesized by Dr. Chun-Cheng Lin's laboratory at National Tsing Hua University (Hsinchu, Taiwan) and dissolved in DMSO. The structure and synthetic scheme of CCL-34 and CCL-44 were described in [11]. The possibility of LPS contamination in both compounds has been excluded [11].

\section{Cell viability}

DCs were harvested on day 8 and the numbers of viable cells were counted using the trypan blue dye exclusion test. The recovery rate of DC was estimated by dividing the number of harvested $\mathrm{DC}$ by the total number of sorted CD14+ monocytes. The amount of dead cells was estimated by using 7-AAD staining and flow cytometry analysis.

\section{Surface antigen expression on DC by flow cytometric analysis}

Dual-color immunolabelling was performed using fluorescein isothiocyanate (FITC)- and phycoerythrin (PE)-conjugated monoclonal antibodies (mAbs). The mouse anti-human mAbs IgG1:FITC/mouse IgG1:PE, and appropriate isotype controls were purchased from Serotec (Oxford, U.K.) and used for DC characterization as follows: anti-CD14 (for IgG-FITC), anti-CD1a-PE, antiCD80-PE, anti-CD86-PE, anti-CD83-PE, anti-HLA-DR -PE and anti-DC-SIGN-PE. Cells were incubated with saturating concentrations of $\mathrm{PE}$-conjugated $\mathrm{mAbs}$ or primary $\mathrm{mAbs}$ followed by IgG-FITC at $4^{\circ} \mathrm{C}$ for $30 \mathrm{~min}$. After washing twice with PBS, $1 \times 10^{6}$ cells were applied to a FACS caliber flow cytometer (BD Biosciences, San Jose, CA). Data were collected and analyzed using CellQuest Software (BD Biosciences).

\section{Phagocytosis assay}

A quantitative measurement for the phagocytic capacity of DCs was performed by allowing the cells to engulf large ( $2 \mu \mathrm{m}$ in diameter) immunofluorescent latex beads (FITC-conjugated dextran-modified latex bead, Sigma). Cells were harvested and allowed to phagocytose the beads for one hour at 4 or $37^{\circ} \mathrm{C}$. The cells were washed for three times and then subjected to flow cytometer analysis. The quantity of phagocytosis by DCs 
is represented as percentages of FITC-positive cells. Data were normalized to phagocytosis obtained with untreated DCs at $4^{\circ} \mathrm{C}$.

\section{Morphological observation}

Harvested DC were centrifuged onto microscope slides by a Cytospin ${ }^{2}$ centrifuge (Shandon Inc, Pittsburgh, PA), stained with Liu's solution, and observed under light microscopy (Olympus, Tokyo, Japan). Photographs were taken with a digital camera equipped on microscopy.

\section{Allogeneic naive $\mathbf{T}$ cell proliferation}

To purify $\mathrm{CD}^{+} \mathrm{CD} 45 \mathrm{RA}^{+} \mathrm{T}$ cells, nonadherent cells from culture of isolated mononuclear cells were used. Naive T cells were enriched with a CD4 ${ }^{+} \mathrm{CD} 45 \mathrm{RA}^{+} \mathrm{T}$ cell isolation kit (Miltenyi Biotec) using a MiniMACS system with magnetic Abs by a negative selection technique. Monocyte-derived DC were harvested and irradiated (3,000 cGy) with $6 \mathrm{MeV}$ X-rays generated by a linear accelerator $\left(\right.$ Clinac $^{\circledR}$ 1800, Varian Associates, Inc., CA) at a dose of $4.0 \mathrm{~Gy} / \mathrm{min}$ in a single fraction. Full electron equilibrium was ensured for each fraction by a parallel plate PR-60C ionization chamber (Capintel, Inc., Ramsey, NJ, USA). Thirty Gray-irradiated DC were incubated with $1 \times 10^{6}$ allogeneic naive T cells at ratios of $1: 10$ or $1: 20$ for 5 days, after which $10 \mu \mathrm{M}$ 5-bromo-2-deoxyuridine (BrdU) was added to T cell cultures for $18 \mathrm{~h}$. The cells were then collected and the incorporated BrdU was detected using flow cytometry.

\section{Detection of cytokines produced by DC and stimulated allogeneic naive $T$ cells}

For human DC, the levels of IL-12 $2_{\mathrm{p} 70}$ and IL-10 in the DC supernatant as well as interferon- $\gamma($ IFN- $\gamma)$ in the stimulated allogeneic T-cell supernatant were measured using ELISA kits purchased from $\mathrm{R} \& \mathrm{D}$ Systems according to the manufacturer's instructions. The detection limits for cytokines were $5.0-8.0 \mathrm{pg} / \mathrm{mL}$. For mouse DC, the IL-12p70 secreted by DC was measured using ELISA kit purchased from R \& D systems, Vienna, Austria).

\section{IL-12 produced by maturated DC from TLR4- defective and wild-type mice}

To clarify whether CCL-34 modulate DC maturation via a TLR4-dependent manner, bone marrow-derived immature DC prepared from the TLR4-defective $\mathrm{C} 3 \mathrm{H} / \mathrm{HeJ}$ and wild-type $\mathrm{C} 3 \mathrm{H} / \mathrm{HeN}$ mice were isolated and cultured as described previously [22]. The growth medium contains $20 \mathrm{ng} / \mathrm{ml} \mathrm{rmGM}-\mathrm{CSF}$ and $20 \mathrm{ng} / \mathrm{ml} \mathrm{rmIL-4}$ for promoting DC differentiation. After incubation for 3 days, the suspended cells were removed and fresh growth medium was added to the adherent cells, which were maintained for an additional 3 days. Then suspension and loosely adherent cells were harvested for subsequent treatment with vehicle, CCL-34 or LPS for $24 \mathrm{hr}$, and assessed for IL-12 production using ELISA assays as previously described.

\section{DC antigen presentation in vivo and analysis of antigen-specific CTL}

For DC antigen presentation in vivo, immunocompetent $\mathrm{Balb} / \mathrm{c}$ mice were treated by intraperitoneal injection of vehicle, CCL-34 or CCL-44 for 3 consecutive weekly dosage of $5 \mathrm{mg} / \mathrm{kg}$. Mice were subcutaneously injected with doxorubicin $(5 \mu \mathrm{M}$ for $24 \mathrm{~h})$ -treated syngeneic colorectal cancer CT26 cells. After one week, the mice were implanted with live CT26 cells at flank and the draining popliteal lymph nodes were removed after tumor grew to greater than $3 \mathrm{~mm}$ in diameter. Single cell suspension prepared from these draining lymph nodes were further challenged by lysates of untreated and doxorubicin-treated CT26 cells for $24 \mathrm{~h}$ and then subjected to analysis for antigen-specific CTL. Toward this end, the expression of surface molecule CD8 and the staining for intracellular IFN- $\gamma$ was analyzed by flow cytometry.

\section{Statistical analysis}

The results were expressed as mean \pm standard error of mean (SEM). Comparison in each experiment was performed using one-way analysis of variance followed by Dunnett's test, and a $p$ value of less than 0.05 was considered statistically significant.

\section{ACKNOWLEDGMENTS}

Funding sources are MMH-E-102-13 from Mackay Memorial Hospital, and NSC-99-2320-B-010-009-MY3 from National Science Council, Taiwan.

\section{CONFLICTS OF INTEREST}

The authors declare no conflicts of interest.

\section{REFERENCES}

1. Banchereau J, Steinman RM. Dendritic cells and the control of immunity. Nature. 1998; 392:245-252.

2. Jonuleit H, Kuhn U, Muller G, Steinbrink K, Paragnik L, Schmitt E, Knop J, Enk AH. Pro-inflammatory cytokines and prostaglandins induce maturation of potent immunostimulatory dendritic cells under fetal calf serumfree conditions. Eur J Immunol. 1997; 27:3135-3142. 
3. Kato T, Yamane H, Nariuchi H. Differential effects of LPS and CD40 ligand stimulations on the induction of IL-12 production by dendritic cells and macrophages. Cell Immunol. 1997; 181:59-67.

4. Cella M, Scheidegger D, Palmer-Lehmann K, Lane P, Lanzavecchia A, Alber G. Ligation of CD40 on dendritic cells triggers production of high levels of interleukin-12 and enhances T cell stimulatory capacity: T-T help via APC activation. J Exp Med. 1996; 184:747-752.

5. Labeur MS, Roters B, Pers B, Mehling A, Luger TA, Schwarz T, Grabbe S. Generation of tumor immunity by bone marrow-derived dendritic cells correlates with dendritic cell maturation stage. J Immunol. 1999; 162:168-175.

6. Evans JT, Cluff CW, Johnson DA, Lacy MJ, Persing DH, Baldridge JR. Enhancement of antigen-specific immunity via the TLR4 ligands MPL adjuvant and Ribi.529. Expert Rev Vaccines. 2003; 2:219-229.

7. Thompson BS, Chilton PM, Ward JR, Evans JT, Mitchell TC. The low-toxicity versions of LPS, MPL adjuvant and RC529, are efficient adjuvants for CD4+ T cells. J Leukoc Biol. 2005; 78:1273-1280.

8. Garcon N, Chomez P, Van Mechelen M. GlaxoSmithKline Adjuvant Systems in vaccines: concepts, achievements and perspectives. Expert Rev Vaccines. 2007; 6:723-739.

9. Alderson MR, McGowan P, Baldridge JR, Probst P. TLR4 agonists as immunomodulatory agents. J Endotoxin Res. 2006; 12:313-319.

10. Dubensky TW, Jr., Reed SG. Adjuvants for cancer vaccines. Semin Immunol. 2010; 22:155-161.

11. Hung LC, Lin CC, Hung SK, Wu BC, Jan MD, Liou SH, Fu SL. A synthetic analog of alpha-galactosylceramide induces macrophage activation via the TLR4-signaling pathways. Biochem Pharmacol. 2007; 73:1957-1970.

12. Lin YS, Huang LD, Lin CH, Huang PH, Chen YJ, Wong FH, Lin $\mathrm{CC}, \mathrm{Fu} \mathrm{SL}$. In vitro and in vivo anticancer activity of a synthetic glycolipid as Toll-like receptor 4 (TLR4) activator. J Biol Chem. 2011; 286:43782-43792.

13. Pantel A, Cheong C, Dandamudi D, Shrestha E, Mehandru S, Brane L, Ruane D, Teixeira A, Bozzacco L, Steinman RM, Longhi MP. A new synthetic TLR4 agonist, GLA, allows dendritic cells targeted with antigen to elicit Th1 T-cell immunity in vivo. Eur J Immunol. 2012; 42:101-109.
14. Svajger U, Pohleven J, Kos J, Strukelj B, Jeras M. CNL, a ricin B-like lectin from mushroom Clitocybe nebularis, induces maturation and activation of dendritic cells via the toll-like receptor 4 pathway. Immunology. 2011; 134:409-418.

15. Pajak B, Garze V, Davies G, Bauer J, Moser M, Chiavaroli C. The adjuvant OM-174 induces both the migration and maturation of murine dendritic cells in vivo. Vaccine. 2003; 21:836-842.

16. Okamoto M, Furuichi S, Nishioka Y, Oshikawa T, Tano T, Ahmed SU, Takeda K, Akira S, Ryoma Y, Moriya Y, Saito M, Sone S, Sato M. Expression of toll-like receptor 4 on dendritic cells is significant for anticancer effect of dendritic cell-based immunotherapy in combination with an active component of OK-432, a streptococcal preparation. Cancer Res. 2004; 64:5461-5470.

17. Lee TY, Kim YH, Yoon SW, Choi JC, Yang JM, Kim CJ, Schiller JT, Sung MH, Poo H. Oral administration of poly-gamma-glutamate induces TLR4- and dendritic celldependent antitumor effect. Cancer Immunol Immunother. 2009; 58:1781-1794.

18. Huang LD, Lin HJ, Huang PH, Hsiao WC, Reddy LV, $\mathrm{Fu}$ SL, Lin CC. Synthesis of serine-based glycolipids as potential TLR4 activators. Org Biomol Chem. 2011; 9:2492-2504.

19. Lin YS, Lin CH, Huang LD, Chao T, Kuo CD, Hung LC, Wong FH, Lin CC, Fu SL. The suppression of thoc1 in cancer cell apoptosis mediated by activated macrophages is nitric oxide-dependent. Biochem Pharmacol. 2013; 86:242-252.

20. Mantovani A, Allavena P, Sica A, Balkwill F. Cancer-related inflammation. Nature. 2008; 454:436-444.

21. Huang B, Zhao J, Li H, He KL, Chen Y, Chen SH, Mayer L, Unkeless JC, Xiong H. Toll-like receptors on tumor cells facilitate evasion of immune surveillance. Cancer Res. 2005; 65:5009-5014.

22. Liu SH, Lin CH, Hung SK, Chou JH, Chi CW, Fu SL. Fisetin Inhibits Lipopolysaccharide-Induced Macrophage Activation and Dendritic Cell Maturation. J Agric Food Chem. 2010. 